\title{
Educación Médica. Renovación en la enseñanza de las ciencias morfológicas*
}

\author{
RECAVARREN ARCE Sixto ${ }^{1}$, SALINAS CERQUIN César ${ }^{2}$, ANTUNEZ DE \\ MAYOLO Antonio ${ }^{2}$.
}

${ }^{1}$ Profesor Principal del Departamento de Patología, Universidad Peruana Cayetano Heredia.

${ }^{2}$ Profesor Auxiliar del Departamento de Patología, Universidad Peruana Cayetano Heredia.

*Este artículo fue escrito antes que la Asamblea Universitaria de la Universidad Peruana Cayetano Heredia dejara sin efecto la fusión de los Departamentos de Ciencias Morfológicas y de Patología.

Los avances logrados por la investigación médica, han producido una verdadera revolución en el conocimiento de ese campo y vemos con asombro como verdades o conceptos médicos que parecían inamovibles se han visto de pronto modificados. Los rápidos cambios ocurridos en la medicina, han hecho que en algunas escuelas médicas sus educadores hayan propuesto nuevas vías en la preparación del estudiante, con el objeto de que logre una formación que garantice el adecuado ejercicio de la profesión en la medicina del futuro.

Los tradicionales métodos utilizados en la enseñanza médica han permanecido estables y resistentes al cambio. Sin embargo a partir de la última década algunos educadores médicos demostraron la necesidad de un cambio y lograron en sus escuelas la implantación de nuevos modelos. Los reformistas empezaron por reconocer que es verdaderamente dificultoso determinar el contenido curricular y el diseño de educación médica apropiados para la medicina del futuro. Señalan también que las escuelas de medicina carecen de una organización que permita la aplicación de las reformas propuestas. También comentan sobre la obstinada resistencia al cambio encontrada en algunas autoridades y profesores y sobre los divergentes criterios existentes entre los que creen en el cambio. Estas inconveniencias o problemas pudieron ser resueltos por la profunda convicción de la necesidad de cambio.

No es propósito del presente escrito hacer análisis del Contenido, del Proceso, de la Organización y de los Resultados logrados, en las escuelas de medicina que han optado por el cambio. Ello es tarea fundamental que corresponde a nuestra Facultad, dado que los intentos de reforma curricular han alcanzado hasta ahora escasos logros en nuestra Institución.

El objeto de la presente comunicación es proponer un modelo que cambie los sistemas de enseñanza de las Ciencias Morfológicas, contribuyendo así a su modernización. 
Existen propuestas y experiencia ya publicadas por escuelas médicas que realizaron reformas curriculares. De estas propuestas hay una que es fundamental pues revela el sentido de modernización de las medidas e intención de cambio. La propuesta en referencia considera que los estudiantes de medicina deben recibir desde el inicio de su formación una educación que integre, en los niveles adecuados, los contenidos de cursos básicos con los de cursos que conecten o introduzcan a las clínicas. En universidades con moderno curriculum se enseña por ejemplo en el primer año de medicina, además de Anatomía humana e histología, Patología, Radiología, Inmunología y Microbiología con niveles y contenidos que permiten a los estudiantes adquirir un bagaje de conocimientos que les servirá para comprender los mecanismos patogénicos de las enfermedades, proporcionando a los estudiantes un nivel de preparación ventajosa para iniciar sus prácticas clínicas, incluyendo la interpretación de los cambios semiológicos.

Creemos que la enseñanza integrada de los cursos de las Ciencias Morfológicas en nuestra Facultad daría los mismos beneficios que se han logrado con el sistema de la enseñanza integrada en otras áreas de la medicina.

Así, enseñar integradamente la histología normal con las alteraciones histopatológicas producidas por la enfermedad, daría al estudiante del primer año un conocimiento fundamental para el entendimiento de lo fisiopatológico, preparándolo anticipadamente para la mejor comprensión de síntomas y signos de los pacientes en sus prácticas clínicas iniciales. Del mismo modo la integración de la anatomía normal con la del órgano enfermo, proporcionaría al estudiante anticipados conocimientos para sus prácticas clínicas. Los niveles con que se dé este tipo de enseñanza deberían ser cuidadosamente establecidos.

Proponemos estructurar un modelo de reforma en la enseñanza de la Ciencias Morfológicas en el que se den la modernización de los sistemas de enseñanza, innovación de la implementación tecnológica e integración de la enseñanza de las ciencias morfológicas. En la propuesta también se sugieren medidas para resolver problemas percibidos en esa área:

- Duración excesiva de las asignaturas

- Profesorado no siempre especializado en el campo de la morfología.

- Implementación tecnológica obsoleta.

- Enseñanza estática de la morfología

La propuesta considera:

Acortamiento del período del tiempo asignado a los cursos de Histología y Anatomía Humana por mejor utilización de las denominadas "horas de práctica". Estas asignaturas se han acortado significativamente en las universidades que han reformado su curricula.

- $\quad$ En cuanto al recurso humano, el profesor debe ser un morfólogo; pero, ¿quién es un morfólogo?. Dentro de los especialistas médicos, el patólogo es quien trabaja cotidianamente en el campo de la morfología, ya sea en microscopía por su labor diagnóstica en histopatología, y en la macroscopía por el estudio de especímenes anatómico quirúrgicos resecados y en las necropsias. No hay otro médico más ligado a la morfología que el patólogo. Por tanto la propuesta considera al patólogo. Por tanto la 
propuesta considera al patólogo como el principal profesor de las Ciencias Morfológicas. Por razones similares el cirujano es anatomista calificado.

- En lo referente a implementación tecnológica nos referiremos primero al microscopio, el que con el tiempo ha mejorado significativamente. Así del microscopio monocular, que todavía se usa en las prácticas microscópicas de nuestras Universidad, se pasó el microscopio binocular, el que tiene sustancias mejoras en el estativo y en los sistemas ópticos y de iluminación. Sin embargo, el uso de este microscopio continúa siendo unipersonal y es limitado para los objetivos de la enseñanza. Luego aparecieron los microscopios de doble cabezal o bipersonales que por primera vez permitirían al profesor la enseñanza dirigida de la histología o histopatología. En los últimos años han aparecido los microscopios de múltiple cabezal para uso multipersonal; los hay de 5, 10, 20 ó hasta más cabezales. Tanto el microscopio de uso bipersonal como el de uso multipersonal tienen incorporado en su sistema óptico un puntero fluorescente que permite al profesor la enseñanza dirigida de la histología o histopatología. Con este sistema el profesor señala las estructuras con el puntero, las enseña y discute con los estudiantes, es decir el profesor dirige la práctica. En cambio con el microscopio monocular el estudiante invierte demasiado tiempo en la identificación de las estructura sy muchas veces por inexperiencia tiene dificultades o inseguridad en su identificación. La práctica dirigida, ahora tiempo y permite una mejor enseñanza de la histología o histopatología. Esa es la experiencia obtenida durante el último año en el Departamento de Patología del Hospital Cayetano Heredia, mediante la utilización de microscopio de múltiple cabezal en la enseñanza de microscopio multipersonal es que con un sólo preparado histológico y con un sólo profesor se puede enseñar simultáneamente a 20 ó 40 alumnos.

Otro importante implemento para la enseñanza de la histología o histopatología es el microproyector con luz halogenada, con adecuadas magnificaciones y niveles de resolución.

Otro implemento de gran utilización en la enseñanza moderna de la histología es la cámara de alta resolución. Esta cámara incorporada a un microscopio óptico permite recibir en un monitor, también de alta resolución, la imagen captada por el microscopio con un grado de resolución, la imagen captada por el microscopio con un grado de resolución mejor que el obtenido con el microproyector. Este método permite asimismo la enseñanza de estudiantes por grupos con la ventaja del alto nivel de resolución de la imagen y la grabación de las prácticas en cintas de video, las que serían, si fuera necesario, utilizadas después por el alumno.

Por último la computadora se está empleando también en la enseñanza de la morfología con ingeniosos programas que desafortunadamente tienen la incoveniencia de su alto costo.

En el Departamento de Patología del Hospital Cayetano Heredia, se ha logrado adquirir dos microscopios de cabezal múltiple que vienen funcionando ininterrumpidamente y acaba de adquirirse una cámara de alta resolución. La capacidad de gestión es en estos tiempos indispensables para que los Departamentos logren implementaciones y modernismos. 
La enseñanza de la histología no debe ser estática, no debe limitarse a la mera descripción de las estructuras, debe más bien correlacionársela con la función pero sobre todo debe correlacionársela con las alteraciones estructurales producidas por las enfermedades y sus consecuentes disfunciones. El nivel con que se de esta información a los estudiantes deberá ser cuidadosamente diseñado. Por ejemplo en el glomerulo renal, la discutirse la estructura normal de la membrana basal, deberá informarse al alumno que esa es la estructura que se afecta en las llamadas glomérulo-nefritis y que al enfermarse altera su estructura, mostrándosele al alumno el ejemplo de membrana basal enferma y como la lesión altera la filtración como consecuencia. Al enseñar el epitelio bronquial normal se indicará al estudiante que de ese epitelio se genera el más frecuente de los cánceres pulmonares; al discutir los alveólos pulmonares normales, mostrarles como se llenan de líquido en el edema pulmonar o de exudado en las neumonías. En cada órgano o tejido el alumno recibirá, junto a lo normal, información sobre los cambios patológicos más frecuentes.

De esta forma la enseñanza de la histología se haría más dinámica e interesante para el estudiante, pero sobre todo proveería al estudiante de fundamentos estructurales indispensables en la interpretación y comprensión de los síntomas y signos de los pacientes, facilitando así sus prácticas clínicas iniciales, a las que con los sistemas vigentes llega sin los conocimientos de patología básica.

Por último, las propuestas de modificación de la enseñanza de la Anatomía Humana. En Cayetano Heredia y en otras universidades el curso de Anatomía Humana dura 16 semanas. En Harvard dura 8 semanas y en otras universidades aún menos.

El curso de Anatomía Humana debería abandonar los sistemas detallistas de la anatomía descriptiva o topográfica en favor de un curso que imparta conocimientos anatómicos generales. La utilización de cadavéres formolizados debe ser limitada a la enseñanza del sistema muscular en segmentos previamente disecados por técnicos; el alumno no debe disecar músculos, arterias o nervios, procedimientos que consumen demasiado tiempo. El uso de modernas maquetas anatómicas es muy útil para estos fines y contribuye también a definir los niveles generales del curso.

La propuesta enfatiza el uso de la necropsia y de piezas anatómicas frescas, no fijadas con formol, para la enseñanza de la anatomía humana. Existen ya algunas Universidades que realizan el curso de anatomía en la sala de autopsias. La víscera fresca es de fácil disección y da al alumno la información real de lo que es la estructura macroscópica normal; permite conoce, por ejemplo, el real color y consistencia del hígado, la textura normal de las válvulas cardíacas, el aspecto liso de las coronarias, la crepitación el aire alveolar, los pliegues normales de la mucosa gástrica o intestinal.

La propuesta considera también la enseñanza paralela de órganos enfermos. Así, mostrar al lado de un hígado normal uno cirrótico; frente a coronarias normales, unas arterioesclerótica; o válvulas cardíacas afectadas por fiebre reumática; o riñones empequeñecidos por nefritis; o un útero agrandado por miomas.

Esta información sobre patología macroscópica general le será de gran utilidad al estudiante en sus prácticas clínicas iniciales ya que tendrá un conocimiento estructural básico que el alumno usará en la interpretación de la sintomatología y signología del paciente. 
Los alumnos del primer año de medicina están perfectamente capacitados para recibir este nivel de enseñanza morfológica integral.

\section{REFERENCIAS BIBLIOGRÁFICAS}

1. Centofani M. Envolving Medical Education. Hopkins Medical News. 1992; fall: 1831.

2. Tosteson DC. New pathways is General Medical Education. N Engl J Med 1990; 2: 234-238.

3. León R. Fórmula para mejorar la práctica, la docencia y la investigación médica. Volumen conmemorativo del Hospital Nacional Cayetano Heredia 1988-1993. 1993:1317.

\section{Correspondencia:}

Dr. Sixto Recavarren

Departamento de Patología, Hospital Nacional Cayetano Heredia.

Av. Honorio Delgado s/n

San Martín de Porras.

Lima, Perú. 